\title{
Long Range Research at GEC
}

\author{
- an EPS Associate Member - \\ D. G. Scotter, Wembley
}

Ballistic transport, Channelled electrons, Organic molecules, Biotechnology, Perception

From its earliest beginnings, microelectronics has advanced through progressive decreases in the lateral dimensions of basic components. Dimensions have halved about every three years and if the trend is continued (Fig. 1) devices will be only 10 nanometres across by the year 2000 . In parallel, the technology has broadened in scope having grown out of the initial area of integrated circuits for memory and logic applications, now to challenge several older technologies in applications such as microwave sources, electro-optic displays and imaging devices, while also giving rise to new devices like the solid state laser.

\section{Physics of Very Small Electronic De- vices}

As devices have become smaller, new techniques have had to be adopted for their fabrication. Amongst those recently introduced is the Electron Beam Microfabricater which has demonstrated a capability down to around 10 nanometres and shows promise of being suitable, in association with $\mathrm{X}$-ray lithography, for large-scale production. It is now also possible to reduce layer thicknesses down to around 100 nanometres by using Molecular Beam Epitaxy in which a molecular beam is deposited in an ordered manner on to a substrate, a technique which offers many other advantages including a better control over the doping distribution within the material. These latest advances open the prospect of exploiting quantum phenomena in electronic devices, as for example in the use of quantum well structures in III-V materials for producing solid state lasers, that in turn might be extended to superlattices of II-VI materials with tunable band gaps. There is even the possibility of exploiting interference and diffraction effects thereby giving rise to new concepts in electronic device principles based on optical analogues. These techniques lead us into areas where there is considerable uncertainty over the physical mechanisms which will affect or even dominate the characteristics of very small electronic devices.

One highly specialised example, currently under study, is ballistic transport, the term which describes the phenomenon of carriers traversing the entire base region of a field effect transistor without a collision or scattering event. Such scattering processes normally provide the mechanism for keeping the semiconductor lattice and carriers closely coupled and in thermal equilibrium. In their absence, as in ballistic transport, those carriers which are accele-

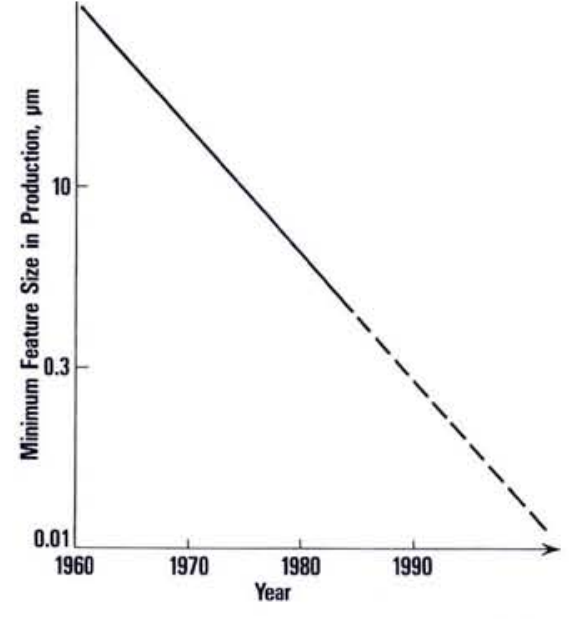

Fig. 1 - Decrease with time of the minimum geometric feature size of integrated circuits in commercial manufacture.

rated, acquire an energy distribution, characteristic of temperatures somewhat above that of the host lattice; i.e. they become hot. A number of non-linear phenomena follow from this effect, contrasting with the linear response which is typical of larger devices. In most devices however, various residual scattering processes still play a role in determining the overall characteristics.

The two principal processes are the interactions among carrier electrons (electronelectron) and between carrier electrons and the vibrational modes of the crystal lattice (electron-phonon). Measurements are already underway on the latter, because of their possible importance in very small electronic devices.

Although in bulk three-dimensional systems, electron-phonon interactions, which are basic to our understanding of electrical resistance and thermal conductivity, are generally well understood, this is not the case in very small electronic devices where we have:

(1) a large surface area in relation to the active volume,

(2) a transport medium that behaves as a two-dimensional electron gas,

(3) the proximity of interfaces with discontinuities in chemical, electronic and elastic properties and

(4) complex three-dimensional vibrational properties of the overall structure.

It is not known to what extent these differences affect the transport but it can be assumed that the effects will become increasingly important as dimensions are reduced further.
To explore electron-phonon scattering in situ we are using a technique known as "frequency crossing phonon spectroscopy", perfected by Professor L.J. Challis of Nottingham University (see Fig. 2a). In its elementary form, a heater produces an acoustic signal with a phonon energy distribution having a black body type of spectrum as indicated in curve 1 of Figure 2 (b). The phonons propagate into a sapphire where at low temperature there is no bulk phonon scattering and the associated thermal conductivity is limited by surface scattering.

The sapphire rod is, however, doped with about $200 \mathrm{ppm}$ of paramagnetic ions of vanadium whose ground state energy level is split by applying a magnetic field (Zeeman effect). Phonons of energy equal to the difference between the two levels are scattered via the electron-phonon interaction leaving a "hole" in the phonon energy spectrum, the frequency of which is proportional to the magnetic field. The depth of the "hole" depends on the strength of the interaction, the width, on both the scattering strength and the strain/magnetic field inhomogeneity broadening. This is, in turn, a function of the relative scattering strengths of impurities

Fig. 2 - Illustration of frequency crossing phonon spectroscopy showing (a) the experimental set-up and (b) the thermal spectra:

1) Heat current spectrum from heater,

2) After modification by 2-D electron gas,

3) Hole burned in spectrum by vanadium doping is tuned to correspond.
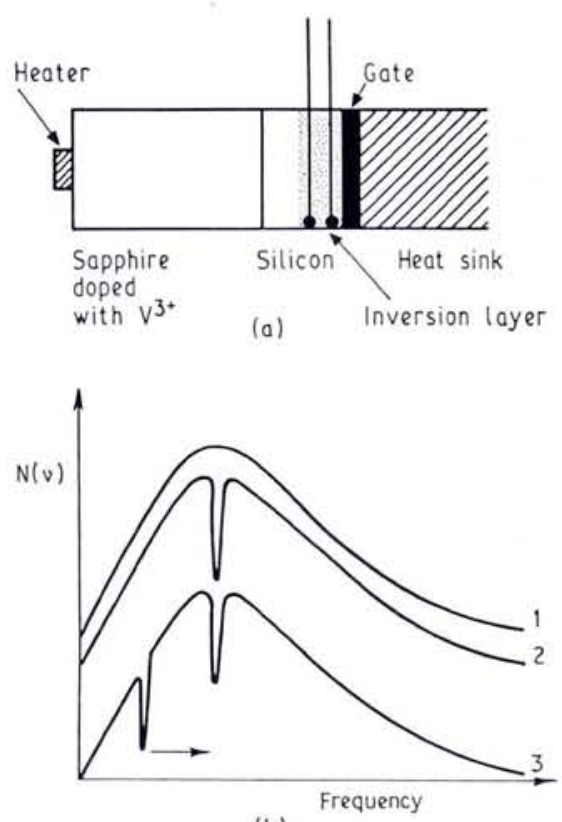

(b) 


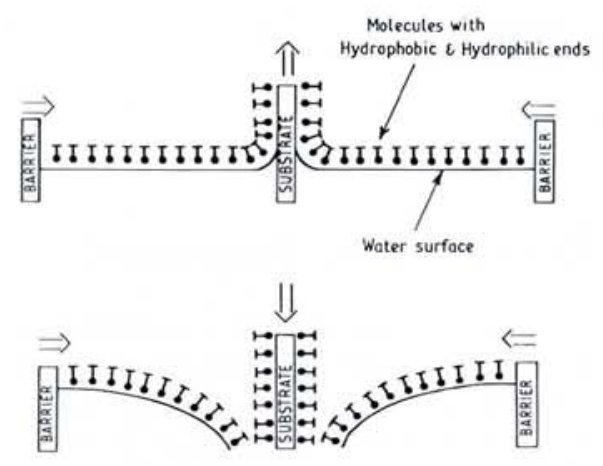

Fig. 3 - Langmuir Blodgett film technique for the transfer of organic films to a substrate.

and the boundaries. The modified phonon spectrum then propagates into a silicon Metal Oxide Semiconductor (MOS) structure which is grown on the sapphire. Within the inversion layer, the active region of the MOS, a second hole will be burnt in the phonon spectrum through electronphonon interactions involving electrons scattered between Landau levels and electrons occupying states within the different silicon conduction band minima. It is this hole which contains the experimental information required.

In order to analyse the second hole, the hole produced by the vanadium ions is tuned by the magnetic field to coincide in frequency (curve 3 ). When this occurs there is an increase in the thermal conductivity as the scattering is then confined to a depleted population in the phonon distribution. Determining the conductivity change under different experimental conditions then allows us to derive the properties of the scattering that has taken place in the MOS inversion layer. Since the characteristics of vanadium doped sapphire are accurately known, we use it as a well-calibrated hole generator for obtaining quantitative data from a range of samples.

We are also exploring the use of this non-destructive technique to analyse the anharmonic scattering from interfaces to give information on their nature. Other experiments with the same apparatus are concerned with transport phenomena such as quantum oscillations in magneto-resistance, and the quantisation effects of phonons propagating along structures having a cross-section comparable with the phonon wavelength 1 ).

Because of the possible importance of $\mathrm{X}$-ray lithography in the manufacture of very small electronic devices, i.e. the superposition of patterns of planar devices by $\mathrm{X}$-rays, and because present day $\mathrm{X}$-ray sources are barely adequate for this role, we are paying attention to the physics involved in the production of suitable X-ray beams. Of particular interest is the emission of $\mathrm{X}$-rays from channelling electrons in single crystals (see R. Wedell, Europhysics News, 12 (1981) 12).

The basic idea, suggested by J.C.H.
Spence and J.M. Cowley, is to use the same crystal to both channel the incident electron beam and provide through Bragg diffraction a "resonant cavity" for the $X$-rays. In a typical set-up, an electron beam is channelled between the $(100)$ planes of a cubic crystal such as diamond. $\mathrm{X}$-rays are emitted at right angles and diffracted by the lattice to give a beam of high intensity and narrow energy width.

Our aim is to obtain a complete understanding of the production of channelling radiation in such a system. This requires the solving of the coupled equations for the electron and photon fields, taking into account the interactions with the crystal. This is a complicated theoretical investigation and, although we cannot be certain that we have considered every important factor, the initial results are promising. Certain ideas for achieving population inversion of the transverse energy levels of the channelled electrons are also being explored, for which experimental work will be needed to confirm whether or not they are valid.

\section{Electronic properties of Organic Mole- cules}

It is not yet known what factors will determine the ultimate limits to the operation of very small electronic devices, but it is worth noting that biological processes involving electron transfer operate on the nanometre scale and at readily accessible temperatures. We are a long way in electronic device technology from approaching such dimensions or realising comparable complexities of structure and action, and it seems reasonable to suggest that organic molecules may play some future role in electronic devices. There is, in any case, a need for better theories to account for their observed electrical and optical properties.

As a means of producing films of organic molecules only nanometres thick, in a form compatible with electronic device structures we have been giving renewed attention to the Langmuir-Blodgett technique. The organic molecules of interest are dissolved in a suitable solvent (such as chloroform) and a drop of the solution is added to water within a confining barrier
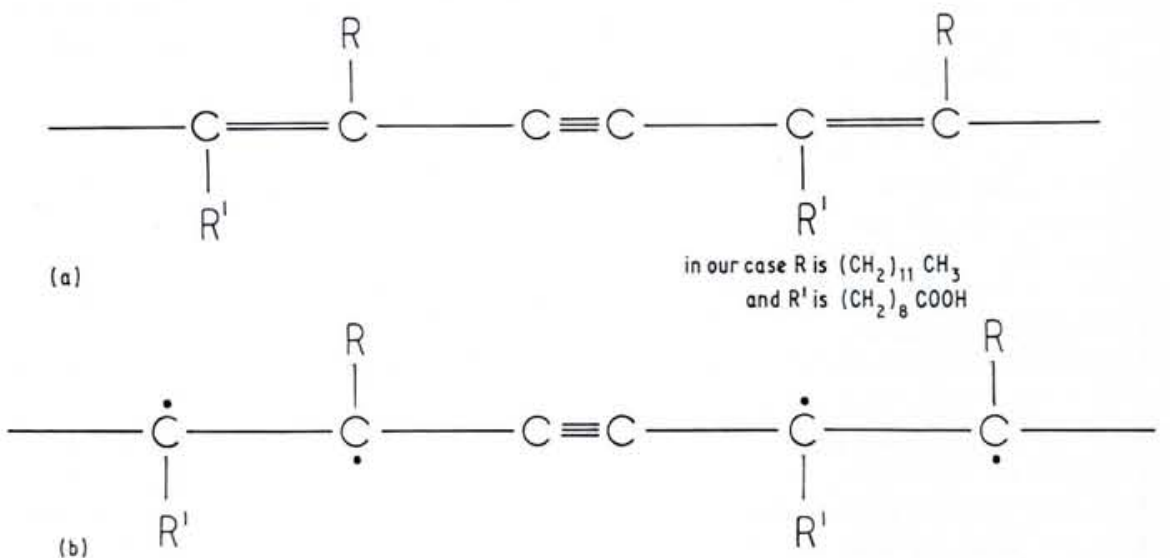

Fig. 4 - Structure of a polydiacetylene - (a) Ground state, (b) Excited, charge carrying hole state. and allowed to spread over the surface. As the solvent evaporates, the barrier is drawn inwards around the molecules, compressing them into a continuous layer of minimum thickness. A substrate with appropriate surface preparation, e.g. a glass slide with a flash of aluminium, is then passed upwards and downwards through the airliquid interface, a monolayer of molecules being transferred on to the substrate surface, at each passage (Fig. 3). Continuity of the monolayer is maintained as molecules are removed, by progressively reducing its area. In practice this is done by moving the barrier under the control of an electronic feedback mechanism which keeps films of uniform packing density can be prepared.

Most of our work has been based on a polydiacetylene; pentacosa-10, 12-diynoic acid which, like most polydiacetylenes, shows high anisotropy in its electrical and optical properties measured parallel and perpendicular to the polymer chain direction. We have recently discovered a dipping procedure which ensures that the polymer chains are aligned with each other in the plane of the Langmuir-Blodgett film which allows us to study the electrical and optical properties in more detail and provides a basis for exploitation.

The electronic properties of polydiacetylene that are associated with a particular arrangement of single double and triple bonds can be analysed using simple bandstructure terms ${ }^{2}$ ), with band parameters derived from experimental data on the geometry of the polymer and its band gap. Unlike the situation in conventional semiconductors, where the crystal structure may be assumed to be independent of the degree of electronic excitation, the polymer backbone can relax significantly when electrons are excited into the conduction band. Indeed, the polymer chain has a crystal structure closely linked to the electronic excitation levels as bond lengths are strongly dependent upon the number of charges involved in each bond. Triple bonds are shorter than double which in turn are shorter than single bonds, and it is the the surface tension constant. In this way 
loosest bound electrons which largely determine the relative bond lengths, as is illustrated in simplified form in Fig. 4.

Theoretical estimates show that the lowest lying excited states involve the loosely bound electrons and are accompanied by a redistribution of bond lengths giving rise to a modification of the structure, see Fig. 4 (b). In practice, the deformation is spread over several atoms but this simple picture is qualitatively correct. In any case, for such excitations to move, it is clear that there must be movement of the atoms. The mobility of the excited electron carriers is affected by the need to move massive atoms and may be limited, at high speeds, by their inability to follow the very rapid electron motions. This may be the explanation for the curious experimental results which show a high initial drift mobility for excited states but saturating at the velocity of sound.

\section{Other Fields of Interest}

Whereas research on the physics of very small electronic devices and the study of electronic properties of organic molecules are the two main programmes of the Long Range Research Laboratory of the GEC, there are two smaller programmes. One is in biotechnology where we are interested in the exact mechanisms whereby electrons taking part in biochemical reactions transfer to electrodes, and vice versa. We see an understanding of this as being crucial to any integration of biotechnology and electronics. The other is in perception where we have already advanced the theory of monocular depth perception from optical flow $^{3}$ ) and are considering extending the studies to higher level visual processing.

Collaboration with other research workers within the GEC and at other research institutions, especially universities, is a particularly strong feature of our activities. Within the GEC there are many other research programmes which have a significant basic research content, but which, in most cases, have clearly identified and specific commercial objectives. There are no such specific objectives for the programmes of the Long Range Research Laboratory, but all four are are on topics where we expect scientific advances to occur over the next few years and those advances are likely to have commercial implications for the Company. By participation in the scientific research we shall be aware of those advances as soon as they occur and be ready to respond to them.

\section{REFERENCES}

1. Kelly M.J., Thermal Anomalies in Very Fine Structures, J. Phys. C 15 (1982) L 969-973.

2. Movaghar B. and Cade N., Spontaneous Localisation of Excitations in Polydiacetylenes, J. Phys. C 15 (1982) L 807-813.

3. Buxton B.F. and Buxton H., Monocular Depth Perception from Optical Flow by Spacetime Signal Processing, to be published in Proc. Roy. Soc.

\section{SUPERCONDUCTIVITY IN ORGANIC SOLIDS}

\section{Jérome and K. Bechgaard, Orsay}

(Lab. de Physique des Solides, Université Paris-Sud)
Almost all the molecular conductors exhibiting superconductivity that have been found so far are derived from the molecule tetramethyltetraselenafulvalene (called TMTSF). When TMTSF, dissolved in an appropriate solvent containing a salt of a monovalent anion $\mathrm{X}$, is oxidized on a platinum anode, high purity crystals of composition $\mathrm{TMTSF}_{2} \mathrm{X}$ separate directly on the electrode according to the reaction:

\section{2n. TMTSF $+n X^{-} \stackrel{-n \cdot e^{-}}{\rightarrow}\left(\text { TMTSF }_{2} X\right)_{n}$}

In the triclinic crystal, the nearly planar TMTSF units are stacked in a zig-zag pattern along the a-axis (Fig. 1). $X$ is typically a monovalent inorganic anion such as $\mathrm{PF}_{6^{\prime}}$ $\mathrm{ClO}_{4}^{-}$etc. and plays an essential role in the partial filling of the valence band, but otherwise does not contribute directly to electron transport. The anions do, however, in some cases affect the electronic properties of the $(\mathrm{TMTSF})_{2} \mathrm{X}$ series via order-disorder phase transitions at low temperature. The conduction band derived from the overlapping $\pi$-type molecular orbitals is half filled rather than quarter filled as inferred from the $2: 1$ stoichiometry of the salt. This occurs due to the triclinicity
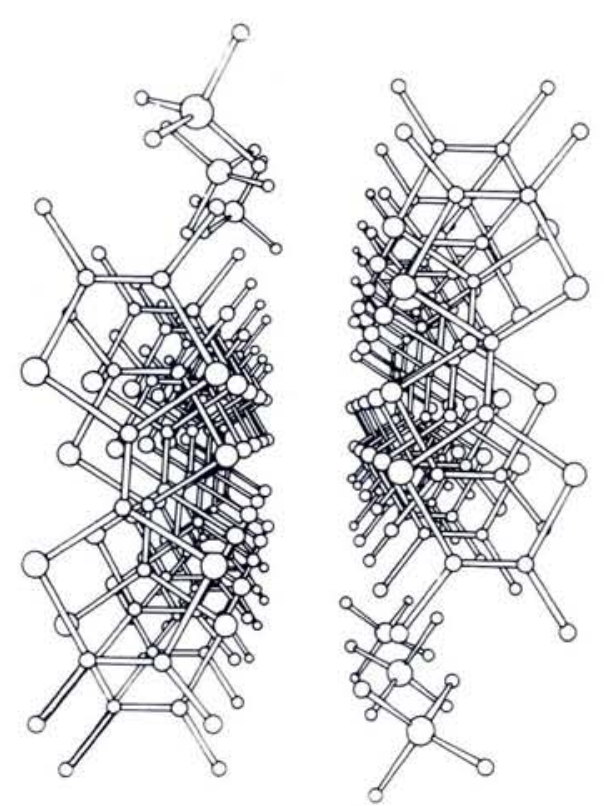
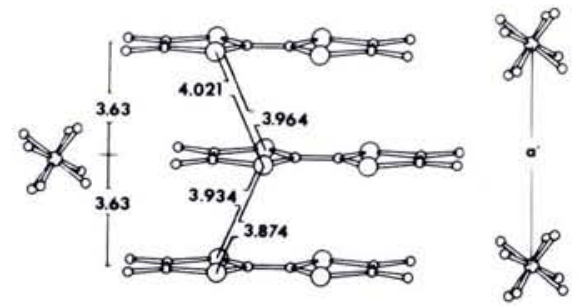

28
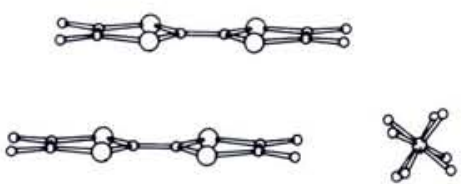

$\int_{0}^{\infty} 8$
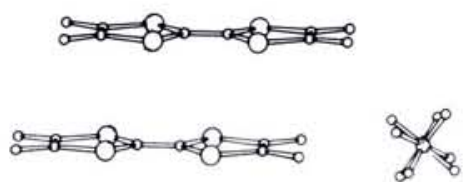

Fig. 1 - View of (TMTSF) ${ }_{2} \mathrm{ReO}_{4}$ along the stacking direction, a-axis (left). The $\mathrm{ReO}_{\overline{4}}$ ions show alternative order along the a-axis $(T<180 \mathrm{~K})$ leading to a 2 a potential. Side-view of $(T M T S F)_{2} \mathrm{CIO}_{4}$ (right). The dimerization is clearly visible in the Se-Se intermolecular contacts (after Thorup et al.). 